\title{
A 2.5D APPROACH TO SKIN WRINKLES SEGMENTATION
}

\author{
Etienne Decencière ${ }^{\llbracket, 1}$, Amira Belhedi ${ }^{1}$, Serge Koudoro ${ }^{1}$, Frédéric Flament ${ }^{2}$, \\ Ghislain François ${ }^{2}$, Virginie RuberT ${ }^{2}$, Isabelle PÉcile ${ }^{2}$ AND Julien Pierre ${ }^{2}$
}

${ }^{1}$ MINES Paristech, PSL Research University, Centre for Mathematical Morphology, 35 rue Saint Honoré, 77300 Fontainebleau, France, ${ }^{2}$ L'Oréal Research and Innovation, 188 rue Paul Hochart, 94550 Chevilly-Larue, France e-mail: etienne.decenciere@mines-paristech.fr

(Received March 22, 2018; revised October 16, 2018; accepted February 11, 2019)

\begin{abstract}
Wrinkles or creases are common structures on surfaces. Their detection is often challenging, and can be an important step for many different applications. For instance, skin wrinkle segmentation is a crucial step for quantifying changes in skin wrinkling and assessing the beneficial effects of dermatological and cosmetic anti-ageing treatments. A $2.5 \mathrm{D}$ approach is proposed in this paper to segment individual wrinkles on facial skin surface described by 3D point clouds. The method, based on mathematical morphology, only needs a few physical parameters as input, namely the maximum wrinkle width, the minimum wrinkle length, and the minimum wrinkle depth. It has been applied to data acquired from eye wrinkles using a fringe projection system. An accurate evaluation was made possible thanks to manual annotations provided by three different experts. Results demonstrate the accuracy of this novel method.
\end{abstract}

Keywords: 3D point cloud, mathematical morphology, skin, wrinkle segmentation.

\section{INTRODUCTION}

With ageing, the human skin changes structurally and becomes more or less wrinkled depending on the combination of factors such as living conditions (e.g. sun exposure, temperature...), life habits (e.g. dietary, tobacco consumption...) and the genetic heritage. Understanding skin wrinkling and improving anti-wrinkle treatments are challenging goals for dermatological and cosmetic research.

Several methods exist for objectively assessing the severity of facial skin wrinkling, either clinically, using standardized photographic scales (Bazin and Doublet, 2007; Bazin and Flament, 2010) or instrumentally. Digital fringe projection systems are gaining popularity, as they provide $3 \mathrm{D}$ point clouds that accurately describe the topography of the skin surface (Jaspers et al., 1999; Rachel, 2003). However, most of the existing solutions to analyze these 3D point clouds are limited to simple global measures that characterize roughness (Whitehouse, 2002). It would be very interesting to segment wrinkles for measuring their length, width, depth and spatial distribution, for instance. To our knowledge, no studies have reported on this subject. Some authors claim to segment skin wrinkles (Jaspers et al., 1999; Luebberding et al., 2014a; b; Akazaki et al., 2002), but the corresponding methods are not accurate enough in terms of segmentation. A higher level of precision is needed for completely describing wrinkles, their alterations and the mechanism of action of cosmetic products or dermatological treatments. Of note, methods that detect wrinkles from on face intensity images (Kwon and Lobo, 1999; Batool and Chellappa, $2012 ; 2015$ ) or on face parallel-polarized images (Cula et al., 2013) are not convenient for our task, as they cannot provide accurate information on their depth.

In the broader field of 3D point cloud analysis, methods for the detection of ridge and valley lines have been previously reported by Ohtake et al. (2004) and by Yoshizawa $e t$ al. (2005). These methods are based on derivatives computation. Given that the structures we are interested in cannot always be characterized by high derivative values, these methods are not appropriate to our goal.

In order to address this issue, a two-and-ahalf dimensional (2.5D) approach was adopted by transforming the $3 \mathrm{D}$ point clouds into $2 \mathrm{D}$ topographic maps that can be considered as range images. This approach has met success in domains such as urban modelling from 3D point clouds (Hernández and Marcotegui, 2009). Now, in the field of range image processing, methods have been proposed to segment images based on first order or second order derivatives discontinuity (Hoffman and Jain, 1987; Hoover et al., 1996). However, these images typically correspond to manufactured objects, made of unions of simple surfaces, far from resembling to human skin surface. Moreover, as previously mentioned, differential approaches seem difficult to use in our context.

Finally, the detection of ridges and valleys on digital elevation models raises challenges similar to 
those of wrinkles detection in range images. Up to our knowledge, these methods resort to differential approaches to model these topographical structures (see for example (Haralick, 1983)), or are based on the simulation of a flow of water along the surface (Soille and Gratin, 1994). We were reluctant to adopt the former approach, as we need to detect small structures in the presence of noise; the latter approach is very specific to drainage networks. A morphological approach seemed more promising.

We propose here a model to define wrinkles on 3D point clouds acquired using a fringe projection technique. This model depends on a few physical parameters (width, length, etc.) and is therefore simple to parameterize. It is implemented using mathematical morphology operators applied to a 2.5D representation of the point cloud. Experiments are reported and validation is performed using manual annotations.

\section{DATA ACQUISITION AND INITIAL PROCESSING}

\section{D ACQUISITION}

Data used in this study was acquired with a dermaTOP fringe projection system developed by Eotech SA (Marcoussis, France). It is based on projecting several fringe patterns on an object and viewing them from a fixed direction. It provides a $3 \mathrm{D}$ point cloud. It is adapted to the analysis of skin area patches of about $3.5 \mathrm{~cm} \times 4.5 \mathrm{~cm}$ including, as examples, crow's feet, forehead wrinkles, wrinkles around the lips, etc. . This paper focuses on the crow's feet region, though the proposed method is not limited to it.

Note that the resulting point clouds included reflection information, which has not been used in this study, as we were only interested by topographic information.

\section{D TO 2.5D TRANSFORM}

The 3D point cloud of the crow's feet region covers a volume of about $45 \mathrm{~mm} \times 35 \mathrm{~mm} \times 20 \mathrm{~mm}$. An example of such a point cloud is presented in Figure 1. Each point $\mathbf{Q}$ of the point cloud can be written as $\mathbf{Q}=$ $(i s, j s, Z$ ), where $Z$ is a real number (corresponding to a depth in microns), $s$ the spatial sampling step of the system (also a real number, in microns), and $i$ and $j$ positive integers. Moreover, for each $(i, j)$ in $\mathbb{Z}^{2}$, there is at most one point in the cloud with abscissa and ordinate equal to $i s$ and $j s$, respectively. That is, the point cloud can be described as a function of a subset of $\mathbb{Z}^{2}$ into $\mathbb{R}$.

Therefore, 3D point clouds can be transformed into 2.5D images by parallel projection without loss of information. A point $\mathbf{Q}$ of coordinates $(i s, j s, Z)$ will be converted into a pixel $x$ of coordinates $(i, j)$. Its corresponding grey level value will be $Z-Z_{\min }$, where $Z_{\min }$ is the lowest depth of the points of the cloud. In other words, the value of each pixel in the $2.5 \mathrm{D}$ image represents the elevation of the point above $Z_{\min }$. In practice, these values are included between 0 and 30000 , and are coded with a 16 bit integer, which limits the loss of information.

Of note, the resulting image might contain missing pixels, i.e. points $(i, j)$ such that there is no point $\mathbf{Q}$ in the point cloud with abscissa and ordinate equal to is and $j s$, respectively. These "holes" are filled in the pre-processing step described below. A 2.5D image example is shown in Figure 1c.

The $2.5 \mathrm{D}$ to $3 \mathrm{D}$ transform is simply the back transform using the same acquisition parameter $s$.

\section{GROUND TRUTH GENERATION}

In order to validate the automatic wrinkle segmentation method described in the following section, three experts were asked to manually mark the wrinkles on 40 point clouds using custom developed software. This number was considered large enough to cover the natural variability of this kind of data in the context of this pilot study. Given that a precise drawing of the contours of each wrinkle appeared impractical, the experts had to draw a broken line along the bottom of the valley-like structures corresponding to the wrinkles. The contrast of the $2.5 \mathrm{D}$ image was adapted by each expert to make the wrinkle visualization possible. This step being expert dependent, a source of variability is introduced into the drawing process. An example is shown in Figure 2. The experts were not asked to mark all the wrinkles of the image, but only those in the crow's feet area. They therefore had to provide a rough region of interest, where all wrinkles were marked. The final region of interest was obtained by taking the intersection of the three regions of interest. Only the wrinkles in this final region of interest have been taken into account.

The variability between experts annotations is used to validate the accuracy of the proposed segmentation method. Among the resulting 40 annotated point clouds, 8 have been used for development and 32 for validation, as described hereafter. 


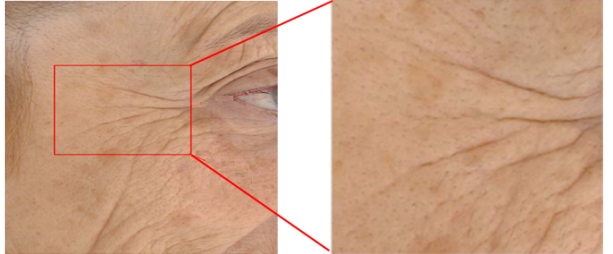

(a) 2D image

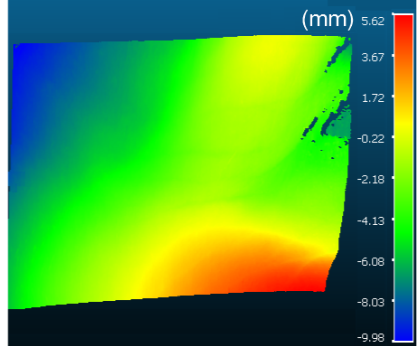

(b) 3D image

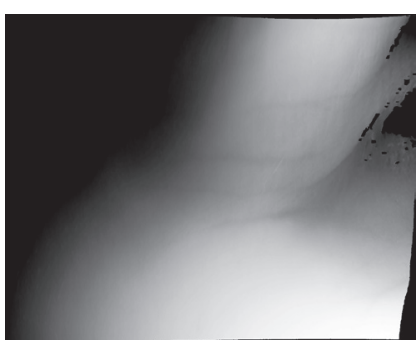

(c) $2.5 \mathrm{D}$ image

Fig. 1. Illustration of point cloud acquistion, and transformation into a 2.5D image: (a) photograph of the crow's feet; (b) 3D point cloud acquired with a fringe projection system; (c) corresponding $2.5 \mathrm{D}$ image (the contrast of this figure is enhanced for better illustration). Here, the sampling step of the point cloud is $35 \mu \mathrm{m}$. The definition of the resulting $2.5 \mathrm{D}$ images is about $1280 \times 1000$ pixels.

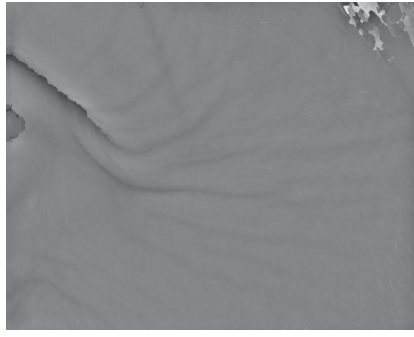

(a) Preprocessed image

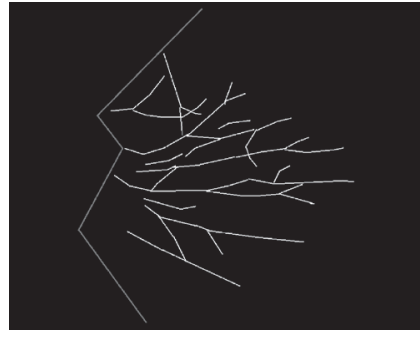

(b) Annotation by expert 1

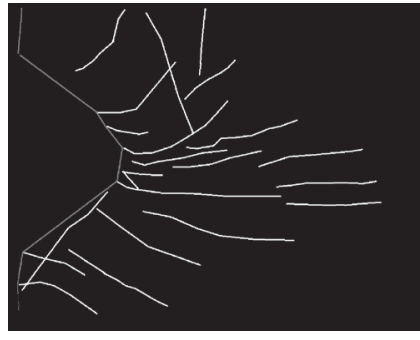

(c) Annotation by expert 2

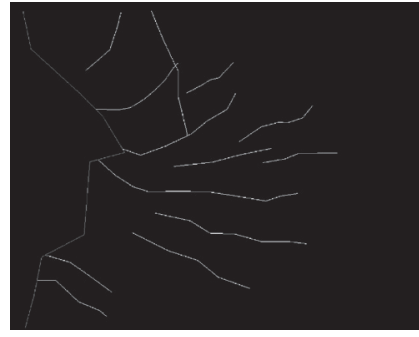

(d) Annotation by expert 3

Fig. 2. Examples of wrinkles annotated by experts on the same point cloud. Wrinkles are marked by bright lines. The region of interest is indicated by a darker line, on the left. Beyond that line, the experts have not marked any wrinkles.

\section{WRINKLE SEGMENTATION}

Our approach to wrinkles segmentation operates on the resulting $2.5 \mathrm{D}$ images. It is based on mathematical morphology.

\section{PRE-PROCESSING}

The pre-processing step aims at filling missing pixels, and removing low frequency components from the surface.

- Missing data interpolation: morphological closings with square structuring elements of increasing size are applied until all empty regions are filled. For each missing pixel, the smallest filling value is kept and the other pixels are left unchanged. The aim of this procedure is to fill the missing pixels in the image, without introducing structures that could be considered as wrinkles.

- Surface flattening by difference between the original image and the result of its convolution with a large Gaussian kernel $G(\sigma)$. $\sigma$ is set to $3,5 \mathrm{~mm}$, that is, approximately three times larger than the width of the widest wrinkles. This operation preserves "small" details, including wrinkles, while removing low frequencies from the surface.

This pre-processing step is illustrated in Figure 3. Note that details, such as vellus hair, small wrinkles and even sweat pores, are much more visible after application of this preprocessing. Of course, this information was present in the original image, but viewing it was difficult given the hight dynamic range of the skin surface in the $2.5 \mathrm{D}$ image. These structures make wrinkle segmentation more difficult.

\section{WRINKLE MODEL}

Wrinkles are surface skin features that appear as elongated valley-like structures. They vary significantly in terms of length, width, depth and orientation, but tend to be globally linear. We therefore model them as straight valleys of minimal length $L_{\text {min }}$, maximal width $W_{\max }$, minimal depth at its center $D c_{\min }$ and minimal depth at its borders $D b_{\min }$. In the current application, the following values were determined empirically, based on the observation of the images: $L_{\min }=2.1 \mathrm{~mm}, W_{\max }=1.1 \mathrm{~mm}, D c_{\min }=1.75 \mathrm{~mm}$ and $D b_{\min }=0.6 \mathrm{~mm}$. Note that these parameters are physical, and therefore are not sensor-dependent or volunteer-dependent. 


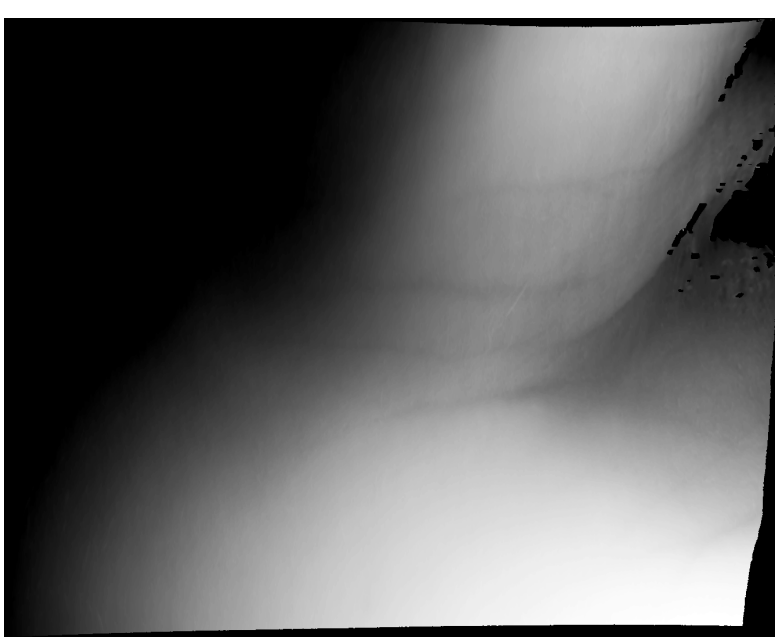

(a) $2.5 \mathrm{D}$ image

Fig. 3. Illustration of the pre-processing method. Left: 2.5D image. Right: result of hole-filling and surface flattening.

\section{IMPLEMENTING THE WRINKLE MODEL}

In order to segment the wrinkles, morphological openings and closings with segments of length $\lambda$ and orientation $\alpha$ are used, respectively denoted $\gamma_{(\lambda, \alpha)}$ and $\phi_{(\lambda, \alpha)}$. The algorithm proposed by Soille et al. (Soille et al., 1996) is used, which leads to an efficient implementation.

Let $I$ be the original 2.5D image. In order to detect wrinkles with direction $\alpha$, we first apply a closing with direction $\alpha+\pi / 2$ and size $W_{\max }$. Its residue $I_{c}$, called a top-hat (Meyer, 1977), gives all dark structures that are thinner than $W_{\max }$ in the $\alpha+\pi / 2$ direction:

$$
I_{c}=\phi_{\left(W_{\max }, \alpha+\pi / 2\right)}(I)-I .
$$

In order to select structures which are long enough, we apply to $I_{c}$ an opening of size $L_{\min }$ along the $\alpha$ direction:

$$
I_{o}=\gamma_{\left(L_{\min }, \alpha\right)}\left(I_{c}\right)
$$

To ensure that the corresponding structures are deep enough, a hysteresis threshold is applied to the result, with values $D b_{\text {min }}$ and $D c_{\text {min }}$. Finally, an area opening of size $W_{\max } \times L_{\text {min }}$ allows small detections, typically corresponding to false positives introduced by facial hair, to be removed. The final image is $I_{\alpha}$.

The procedure is repeated for all directions between 0 and $\pi$, with a step of $\pi / 36$. Several angular step sizes were tested. Steps smaller than $\pi / 36$ did not bring any significant improvement to the result. The final output image is the supremum of all $I_{\alpha}$ :

$$
I_{\text {out }}=\bigvee\left\{I_{\alpha} \mid \alpha \in[0, \pi / 36, \ldots, \pi[\} .\right.
$$

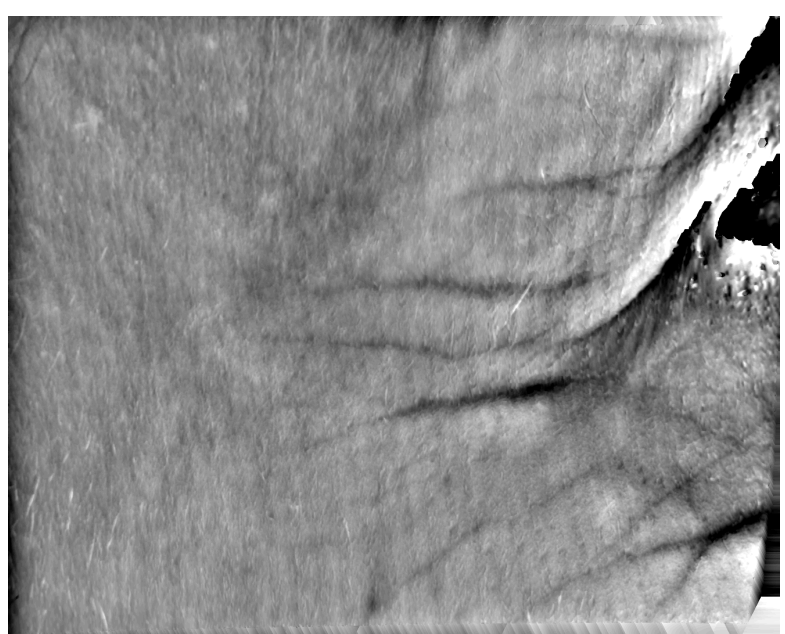

(b) Pre-processing
$I_{\alpha}$, with $\alpha=0^{\circ}$, is shown in Figure 4. The resulting image (see Figure 4c) is a wrinkles map where the value is 0 when there are no skin wrinkles, and the depth of the corresponding wrinkle elsewhere. In order to obtain a binary mask, we simply threshold this image above zero. More examples are shown in Figure 6, after binarization.

Of note, using path operators (Heijmans et al., 2005; Morard et al., 2014) to solve this problem was also considered, but they were needlessly flexible for this application, and computationally more expensive. Indeed, the presented method takes a few seconds to process an image. In some cases, when the image contains large empty regions, the missing data interpolation step is the most time consuming. In any case, even if there is margin for speeding-up this method, the fact is that acquisition takes longer than processing. Therefore, the proposed method meets the user needs from this point of view. We will evaluate the quality of the results in the following section.

\section{VALIDATION}

The data set used for validation corresponds to 32 point clouds of crow's feet acquired in vivo with the fringe projection system. For each point cloud, the corresponding ground truth is generated.

In the following lines, the experimental protocol is presented and its results discussed. 


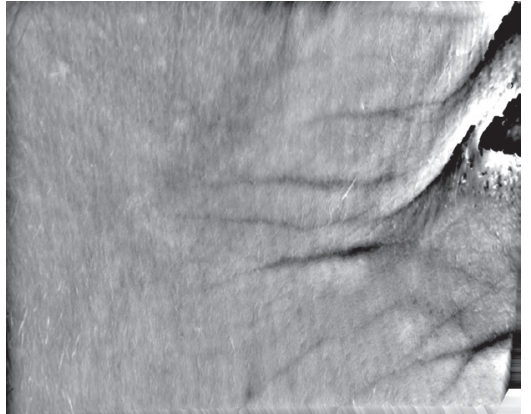

(a) Pre-processing

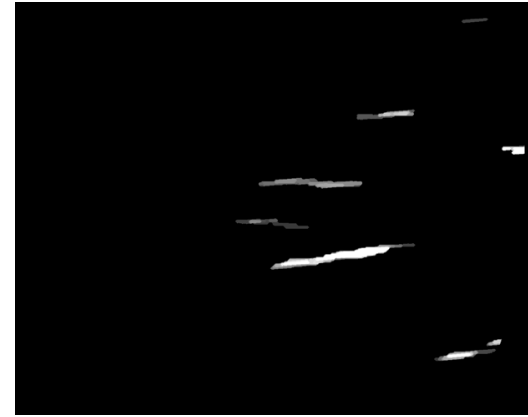

(b) $I_{\alpha}\left(\alpha=0^{\circ}\right)$

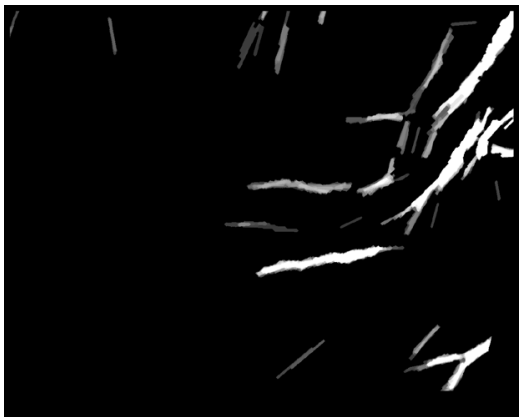

(c) after last iteration

Fig. 4. Illustration of wrinkles segmentation. The original image is shown in Figure 1c. (a) Image after preprocessing (the image contrast is enhanced for better illustration); (b) intermediate result after one iteration; (c) final result.

\section{Experimental Protocol}

Classical precision $(P)$ and recall $(R)$ statistics are computed in order to evaluate our wrinkles segmentation method. The computation is performed in the 3D space, between points in the cloud. It is worth noting that the manual annotations correspond only to the wrinkles axis.

For a given point cloud, let $A$ be the set of points of the cloud which have been considered as belonging to wrinkles by our segmentation method. Moreover, let $C$ be the set of points manually marked by an expert (our ground-truth). Among the points of $A$, those whose distance to $C$ is smaller than max $_{\text {dist }}$ are considered to be "correct detections". They make up set $B$. Therefore, $P$ is given by:

$$
P=\frac{\operatorname{Card}(B)}{\operatorname{Card}(A)} .
$$

Here, parameter $\max _{\text {dist }}$ is chosen equal to $\frac{W_{\max }}{2}$, i.e. half of the width of the largest wrinkles.

Similarly, among the points of $C$, those whose distance to $A$ is smaller than a parameter $\delta_{a}$ are considered to be "correctly detected". They make up set $D$. Therefore, $R$ is given by:

$$
R=\frac{\operatorname{Card}(D)}{\operatorname{Card}(C)} .
$$

Parameter $\delta_{a}$ is an estimation of the experts error when drawing the ground-truth. It has been empirically set to $0.16 \mathrm{~mm}$ (i.e. 4.5 pixels)

An example is presented in Figure 5 showing synthetic data to illustrate the computation of $R$ and $P$.

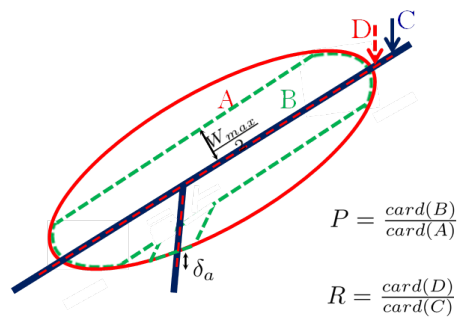

Fig. 5. Illustration of Recall $(R)$ and Precision $(P)$ computation. $A$ and $C$ are point sets corresponding to the segmented points and the annotated ones, respectively. $B$ is defined as the subset of A containing the points whose distance to annotated points is less than $\frac{W_{\max }}{2} . D$ is defined as the subset of $C$ containing the points whose distance to segmented points is less than $\delta_{a}$.

Since the annotations of the three experts are not identical, the variability between their annotations is computed for evaluating the inter-annotator agreement and using it to validate our segmentation. For each annotator, the recall and precision values with respect to the other annotators are computed. The interannotator agreement is defined as the mean value of the obtained $R$ and $P$. In this case, $\max _{d i s}$ is set to $\delta_{a}$.

\section{Results}

The obtained results are shown in Table 1 . In this table, the automatic binary segmentation is compared with the inter-annotator agreement. Recall and precision vary respectively from $21 \%$ to $85 \%$ and from $32 \%$ to $94 \%$, whereas inter-annotator agreement varies from $34 \%$ to $77 \%$. Basically, the automatic segmentation provides similar results as the annotators.

A more detailed analysis shows that the standard deviation of the automatic results is slightly higher than that of the human annotators. Our analysis 
indicates that in some situations (surface globally horizontal; good quality images; numerous wrinkles) the automatic method outperforms human annotators, whereas in more complex situations (noise or artifacts such as facial hair; surface markedly slanted), especially when only a few wrinkles are present, human annotators outperform the proposed method.
Table 1. Mean recall $(R)$ and precision $(P)$ comparison between wrinkles segmentation and inter-annotator agreement.

\begin{tabular}{|c|c|c|c|}
\cline { 2 - 4 } \multicolumn{1}{c|}{} & \multicolumn{2}{c|}{ segmentation } & inter-annotator \\
\cline { 2 - 4 } \multicolumn{1}{c|}{} & Recall (\%) & Precision (\%) & agreement (\%) \\
\hline min & 21 & 32 & 34 \\
\hline max & 85 & 94 & 76 \\
\hline mean & 48 & 62 & 53 \\
\hline std & 16 & 19 & 9 \\
\hline
\end{tabular}
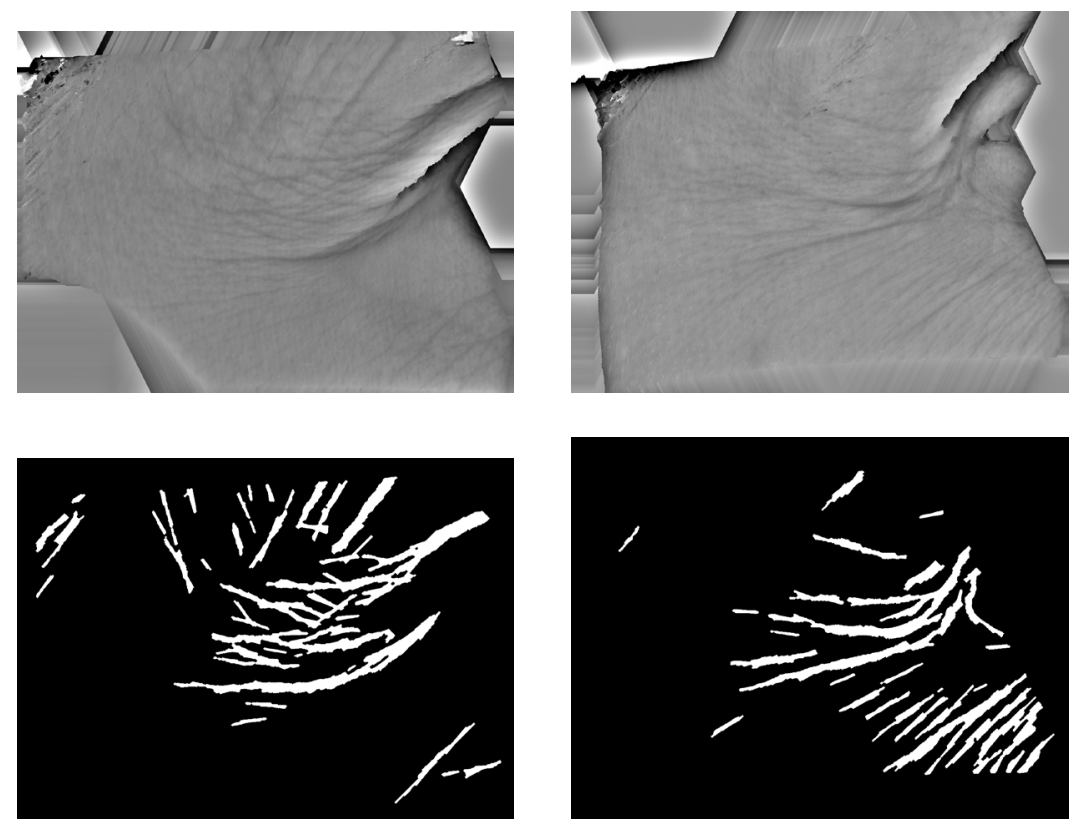

Fig. 6. Results illustration. Contrast has been manually enhanced to improve viewing, when necessary. Top: pre-processed images. Bottom: binary segmentation results.

\section{CONCLUSION}

A wrinkle model and its efficient implementation are presented. It is easy to parameterize as it depends on physical values, such as length and width, skin experts are familiar with. The method is based on a $2.5 \mathrm{D}$ representation of the cloud, and uses morphological openings and closings with segments. Validation was done using data from point clouds corresponding to crow's feet area, on which wrinkles have been manually marked by experts. Such a methodology should pave the way to a precise and neutral assessment of wrinkles severity in medical and cosmetic applications.

This method will be further applied to other facial regions, e.g. forehead wrinkles, wrinkles around the lips, etc. and their progressive changes along the aging process.
The method works as expected when the surface is roughly parallel to the projection plane. As the surface becomes more slanted, the performance of the method decreases. Eventually, if the surface angle with respect to the projection plane approaches $\pi / 2$, the $2.5 \mathrm{D}$ approach becomes inadequate. It should not be too difficult to automatically detect this kind of situation, in order to avoid using the method with inappropriate data. As a perspective, it would be interesting to implement this approach directly in the 3D space, to see if the expected quality improvement would be worth the extra computation load.

The fringe projection acquisition system also provides reflection information. This information is difficult to analyze, as it is the result of complex interactions between light and the skin. However, it 
could be interesting to combine it with the range information in order to improve the quality of our results.

\section{REFERENCES}

Akazaki S, Nakagawa H, Kazama H, Osanai O, Kawai M, Takema Y, Imokawa G (2002). Age-related changes in skin wrinkles assessed by a novel three-dimensional morphometric analysis. BRIT J DERMATOL 147:689-695.

Batool N, Chellappa R (2012). Modeling and detection of wrinkles in aging human faces using marked point processes. In: Fusiello A, Murino V, Cucchiara R, eds., ECCV Workshops (2), vol. 7584 of LECT NOTES COMPUT SC. Springer.

Batool N, Chellappa R (2015). Fast detection of facial wrinkles based on Gabor features using image morphology and geometric constraints. PATTERN RECOGN 48:642-58.

Bazin R, Doublet E (2007). Skin aging atlas, vol. Volume 1. Caucasian type. Med'com.

Bazin R, Flament F (2010). Skin aging atlas, vol. Volume 2. Asian type. Med'com.

Cula GO, Bargo PR, Nkengne A, Kollias N (2013). Assessing facial wrinkles: automatic detection and quantification. SKIN RES TECHNOL 19:1-9.

Haralick RM (1983). Ridges and valleys on digital images. COMPUT VISION GRAPH 22:28-38.

Heijmans H, Buckley M, Talbot H (2005). Path Openings and Closings. J MATH IMAGING VIS 22:107-19.

Hernández J, Marcotegui B (2009). Point cloud segmentation towards urban ground modeling. In: Joint Urban Remote Sensing Event. IEEE.

Hoffman R, Jain AK (1987). Segmentation and Classification of Range Images. IEEE $T$ PATTERN ANAL 9:608-20.

Hoover A, Jean-Baptiste G, Jiang X, Flynn P, Bunke H, Goldgof D, Bowyer K, Eggert D, Fitzgibbon A, Fisher R (1996). An experimental comparison of range image segmentation algorithms. IEEE $T$ PATTERN ANAL 18:673-89.

Jaspers S, Hopermann H, Sauermann G, Hoppe U, Lunderstädt R, Ennen J (1999). Rapid in vivo measurement of the topography of human skin by active image triangulation using a digital micromirror device. SKIN RES TECHNOL 5:195-207.

Kwon YH, Lobo NDV (1999). Age classification from facial images. In: In Proc. IEEE Conf. Computer Vision and Pattern Recognition.
Luebberding S, Krueger N, Kerscher M (2014a). Comparison of validated assessment scales and 3D digital fringe projection method to assess lifetime development of wrinkles in men. SKIN RES TECHNOL 20:30-6.

Luebberding S, Krueger N, Kerscher M (2014b). Quantification of age-related facial wrinkles in men and women using a three-dimensional fringe projection method and validated assessment scales. DERMATOL SURG 40:22-32.

Meyer F (1977). Contrast features extraction. In: Chermant JL, ed., Quantitative analysis of microstructures in materials science, biology and medicine. Caen, France: Riederer Verlag.

Morard V, Dokládal P, Decencière E (2014). Parsimonious Path Openings and Closings. IEEE T IMAGE PROCESS 23:1543-55.

Ohtake Y, Belyaev A, Seidel HP (2004). Ridge-valley Lines on Meshes via Implicit Surface Fitting. In: ACM SIGGRAPH 2004 Papers, SIGGRAPH '04. New York, NY, USA: ACM.

Rachel JD (2003). Skin rejuvenation regimens: A profilometry and histopathologic study. ARCH FACIAL PLAST S 5:145-9.

Soille P, Breen EJ, Jones R (1996). Recursive implementation of erosions and dilations along discrete lines at arbitrary angles. IEEE $\mathrm{T}$ PATTERN ANAL 18:562-7.

Soille P, Gratin C (1994). An Efficient Algorithm for Drainage Network Extraction on DEMs. J VIS COMMUN IMAGE R 5:181-9.

Whitehouse D (2002). Surfaces and their Measurement. New York: Taylor \& Francis Books, Inc.

Yoshizawa S, Belyaev A, Seidel HP (2005). Fast and Robust Detection of Crest Lines on Meshes. In: Proceedings of the 2005 ACM Symposium on Solid and Physical Modeling, SPM '05. New York, NY, USA: ACM. 